OPEN ACCESS

Edited by:

Changiz Mohiyeddini, Northeastern University, United States

Reviewed by:

Serge Brand

Universität Basel, Switzerland

Dianna Theadora Kenny,

University of Sydney, Australia

*Correspondence:

Carolina Inostroza caroinostroza@udec.cl

Specialty section:

This article was submitted to Clinical and Health Psychology, a section of the journal Frontiers in Psychology

Received: 11 January 2018

Accepted: 29 August 2018 Published: 21 September 2018

Citation:

Rincón P, Cova F, Saldivia $S$, Bustos C, Grandón P, Inostroza C, Streiner $D$, Bühring $V$ and King $M$ (2018) Effectiveness of a Positive Parental Practices Training Program for Chilean Preschoolers' Families:

A Randomized Controlled Trial.

Front. Psychol. 9:1751

doi: 10.3389/fpsyg.2018.01751

\section{Effectiveness of a Positive Parental Practices Training Program for Chilean Preschoolers' Families: A Randomized Controlled Trial}

\author{
Paulina Rincón', Félix Cova1, Sandra Saldivia², Claudio Bustos², Pamela Grandón', \\ Carolina Inostroza ${ }^{1,2 *}$, David Streiner ${ }^{3,4}$, Vasily Bühring ${ }^{1}$ and Michael King ${ }^{5}$ \\ ${ }^{1}$ Department of Psychology, University of Concepción, Concepción, Chile, ${ }^{2}$ Department of Psychiatry and Mental Health, \\ University of Concepción, Concepción, Chile, ${ }^{3}$ Department of Psychiatry and Behavioural Neurosciences, McMaster \\ University, Hamilton, ON, Canada, ${ }^{4}$ Department of Psychiatry, University of Toronto, Toronto, ON, Canada, ${ }^{5}$ Division of \\ Psychiatry, Faculty of Brain Sciences, University College London, London, United Kingdom
}

Background: Evidence for the effectiveness of parental training as a strategy for promotion of positive parental practices and prevention of child behavior problems in low and middle income countries is not conclusive. This study aims to assess the effectiveness of a universal positive parental training program designed for this context, "Día a Día" UdeC (c) (“Day by Day" University of Concepción), in Chilean preschoolers' families (3-6 years old children).

Methods: A cluster randomized controlled trial (CRCT) was carried out in 19 preschool education centers. There were two treatment arms: 10 centers (including 178 families) were randomly assigned to the intervention group and nine centers (including 154 families) were assigned to the waiting list control condition. Intervention groups received Day by Day UdeC, a six group sessions program for parents, including two group sessions for preschool educators, focused in affective communication; daily and child-directed play; directed attention; routines and transitions; reinforcement and incentive programs; planned inattention-ignore and time out; and logical consequences. Parental practices, parental satisfaction, and presence of children behavioral problems were examined at two-time points: T1 (4 weeks before intervention) and T2 (5-6 weeks after intervention).

Results: Intention-to-treat analysis shows a reduction in physical punishment and an increase in parental involvement, as well as a reduction in children behavioral problems. A per-protocol analysis revealed an additional effect: increase in observed parental practices.

Conclusion: This CRCT provided evidence for the effectiveness of a parental training program for the promotion of positive parental practices in low and middle income countries. The observed effects of the program in decreasing physical punishment and children's behavioral problems make it a promising strategy for prevention purposes.

Trial Registration: This study was registered under ISRCTN.com (ISRCTN90762146; https://doi.org/10.1186/ISRCTN90762146).

Keywords: promotion, parental practices, parenting program, universal prevention, child behavioral problems, externalizing behavior, preschool children, randomized trial 


\section{INTRODUCTION}

Promoting positive parenting practices appears to be a useful strategy to improve the welfare and psychosocial development of children, and to prevent various psychosocial and mental health difficulties (Herrman et al., 2005). A range of parenting training programs (hereinafter, parenting programs) have been shown to be effective tools for these purposes (Merry and Moor, 2015).

Given the relationship between parenting practices and the development of behavioral problems and disorders in children, several parenting programs have focused on this issue (Furlong et al., 2013; Menting et al., 2013; Forehand et al., 2014). Behavioral problems and disorders during childhood (also called externalizing problems and disorders) are considered among the commonest mental health issues in children and adolescents, resulting in considerable disturbance, especially inside the family and at school. They tend to persist and have negative consequences in several areas of development (Klahr and Burt, 2014).

Although evaluations of parenting programs have focused on the treatment of behavioral problems and disorders, research on prevention is also relevant and shows, in general, good results (Kato et al., 2015; Smedler et al., 2015). However, research has focused on efficacy (studies delivered under optimal conditions with high control from researches, for example, in University clinics) more than on effectiveness (studies conducted in real-word conditions, such as schools and primary care health centers; Streiner, 2002; Merry and Moor, 2015).

In spite of these limitations, the wide-scale implementation of parenting programs of evidence-based parenting programs has the potential to support families and favor the positive psychosocial development of children (Kato et al., 2015).

Our aim was to assess the effectiveness of a group parenting program, implemented at preschool educational centers in low and middle socioeconomic communities in Chile. The purpose of this program, called "Día a Día UdeC" @ ("Day by Day" parenting program developed at University of Concepción, Chile, hereinafter Day by Day), is to strengthen positive parenting practices, specifically focusing on early intervention and prevention of behavioral problems in preschoolers. It was created based on a review of worldwide existing programs which had shown the most promising results ( Kaminski et al., 2008; Gardner et al., 2009). For this research, one version of Day by Day Program assessed in a previous study was modified. This revised version is briefer than the previous one (six sessions as opposed to 10) and includes two working sessions with the educational personnel from the centers in order to make them party to the objectives of the program.

There is more evidence about the effectiveness of target parenting programs (e.g., aimed only at children at risk) rather universal parenting programs. Parenting universal programs had been less studied and they have shown little or no effect (Hiscock et al., 2008; Simkiss et al., 2013). Hiscock et al. (2008) obtained an effect size of 0.22 in the modification of hostile parental practices although these effects were not sustained over time (Bayer et al., 2010). Simkiss et al. (2013) did not see any effect of a 10-session universal training program implemented in low income families. However, the potential impact of effective universal programs, even without large effect sizes, might be relevant both in preventive and promotional aspects (Offord, 2000). For this reason, we decided to develop and assess a universal program. The promotion of positive parenting practices is potentially beneficial for all families and not only for at-risk families. Furthermore, in educational centers, universal programs are easier to implement, are better received, and the risks of stigmatization of selected families are avoided (Merry and Moor, 2015). The universal nature of the program implied special challenges, like the need to develop an intervention as brief as possible in order to encourage the widest participation by families.

Our main hypothesis was that Day by Day Program (experimental group) would result in lower levels of negative parenting practices (inconsistent and punitive practices), and higher levels of positive reinforcement parenting practices and involvement with the child than a waiting list control group. Our secondary hypotheses were that parenting training practice would result in less externalizing behaviors in the children than in the control group, and that participants in the program would show more satisfaction with their parental role and lower levels of depression.

\section{MATERIALS AND METHODS}

\section{Centers}

Twenty preschool educational centers from the Province of Concepción, Chile, were invited to participate in the study. They all accepted the invitation, but one of them finally withdrew (before randomization). Of the 19 participating centers, five belonged to the Integra Foundation, three to the National Council of Kindergarten Schools, six were municipal schools, and five were private subsidized schools. Given the high degree of social stratification of educational centers in Chile, most of the study population were of low socioeconomic background, except in the case of private subsidized centers, where more middle-class families attend.

\section{Participants}

Families from the selected centers having children aged 3 years 0 months to 5 years 11 months were invited to participate (Figure 1). The invitation encouraged the participation of the mother and the father to the Day by Day Program, but only one parent or caregiver per family was considered the main participant for this effectiveness study (hereinafter "participant" or "parent"). Only this main participant was 


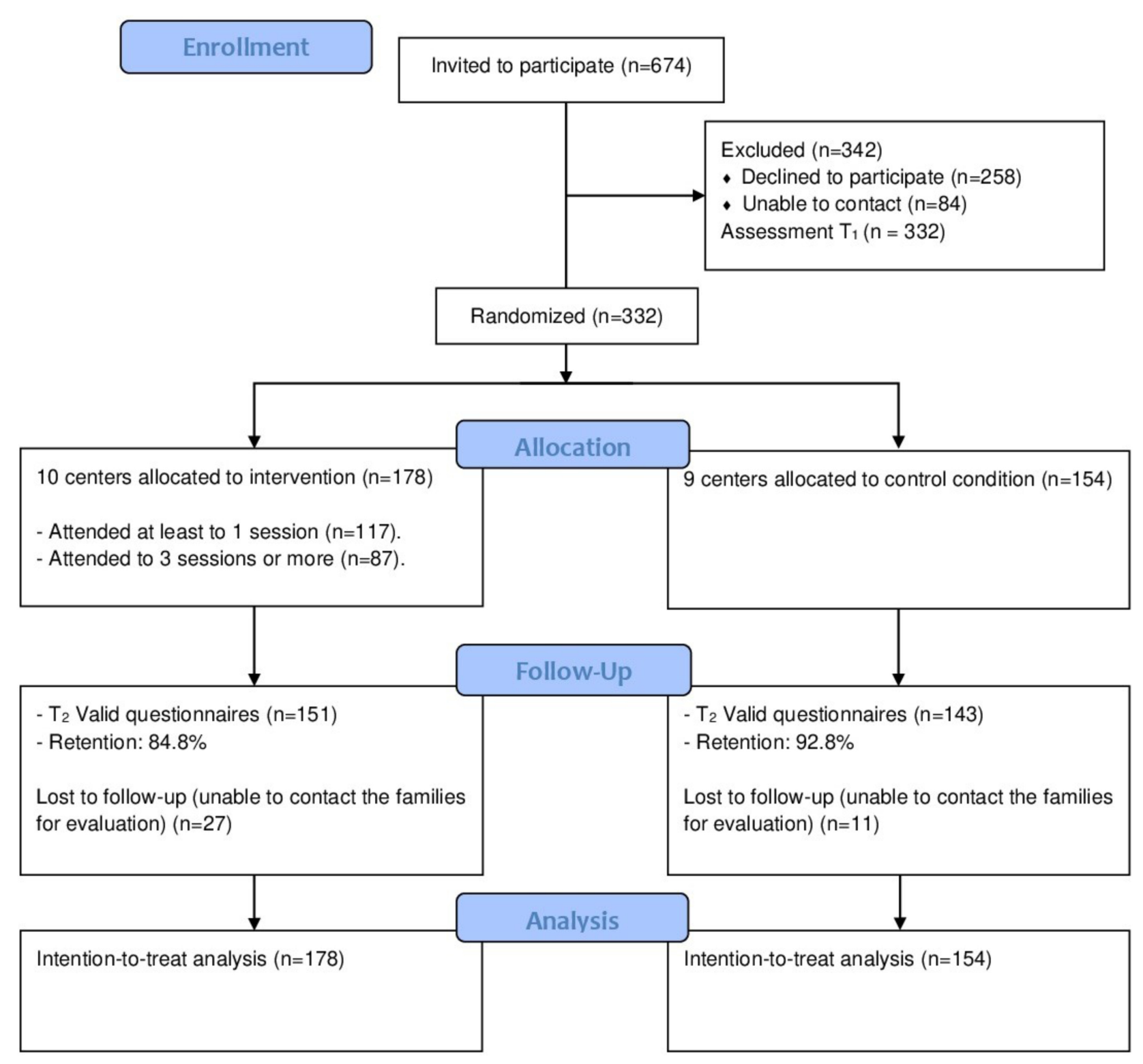

FIGURE 1 | Participants flow diagram.

assessed and included in the analysis. Most of the participants were the children's mothers (87\%) and the remaining were other relatives (fathers, grandmothers, or sisters). In total, 674 families were invited to participate in the research, of whom 416 accepted (declined to participate, $n=258$, a $38 \%$ ). However, not everybody who accepted was able to receive the initial assessment (84 unable to contact). Since these phases were prior to randomization, only the parents or caregivers who received the initial assessment $(n=332)$ were considered as participants of this effectiveness study.

Ten centers containing 178 participants were assigned to the program, corresponding to parents of 94 boys $(52.8 \%)$ and 84 girls (47.2\%) with an average age of 3.7 years
$(\mathrm{SD}=1.0)$; the parents' mean age was 30.6 years $(\mathrm{SD}=6.8)$. Nine centers with 154 participants were assigned to the waiting list condition, corresponding to 81 boys (52.6\%) and 73 girls $(47.4 \%)$ with an average age of 3.9 years $(\mathrm{SD}=1.0)$; their parents were 31.5 years old $(\mathrm{SD}=6.9)$ on average.

The number of participants per cluster ranged from 13 to 23 .

\section{Power and Sample Size}

We sought to demonstrate an effect size at least 0.3 with an assumed intraclass correlation for clustering of 0.01 , and correlation between baseline and post-test of $r=0.5$. To achieve this at $80 \%$ power and a level of 
significance of $0.5 \%, 306$ participants, 153 in each arm, were needed.

\section{Randomization and Masking}

The randomization of the centers was performed after inviting the participants to be part of the research and conducting the baseline assessments. Thus, at the time of the pretest assessment $\left(\mathrm{T}_{1}\right)$, participants and evaluators did not know who would participate in each group. One person from outside the research team was in charge of the entire randomization process using a ballot box and tickets. Block randomization was conducted according to the institutional affiliation of the centers. Each institution of the block, represented by numbers, was added to the ballot box. The first selected numbers, corresponding to half of each block, were assigned to the experimental group (and removed from the ballot box) and the remaining numbers to the control group. Given that three of the four blocks were odd, and that in the final balance there should be 10 experimental centers and nine control centers, the two blocks that should have an additional center assigned to the experimental group and the one that should have an additional control center were raffled.

\section{Training Program}

Day by Day Program contains the following seven components: affective communication; daily and child-directed play; directed attention; routines and transitions; reinforcement and incentive programs; planned inattention-ignore and time out; and logical consequences. As methodological training strategies, the following are employed: participatory analysis of concepts and 19 videos showing daily interactions between one parent and the child, with different degrees of parental practice performance; role-play; rehearsal with children in the session; tasks to be applied at home; self-reports; and readings and videos to be used at home.

The implemented version of the program had six 2-h group weekly sessions. It was completely manualized and was implemented during the months of May-July 2016. It was given by psychologists with more than 5 years of psychology studies who were trained by the research team in a $40-\mathrm{h}$ program (26 face-to-face hours). In order to be able to implement the program, the trained psychologists were required to undergo a performance test.

A number of measures to optimize participation were taken, such as weekly reminder calls, a day care system to allow participants to attend the center with their children, and a system of small rewards for attending (Chacko et al., 2016).

After each session, each facilitator completed a check list regarding the implementation of it, which was registered in an on-line system. The research team conducted weekly face-to-face or online therapist's supervision. In order to assess fidelity to the program, a member of the research team made an onset observation of $10 \%$ of the sessions. Fidelity of the program, measured by adherence to program guidelines, was estimated at $84 \%$.

\section{Variables and Instruments}

The effect of the program was evaluated in three areas: (1) parental practices, (2) parental satisfaction, and (3) children's behavioral problems. Pre-assessment $\left(\mathrm{T}_{1}\right)$ was carried out 4 weeks before the beginning of the program at each center and the post-assessment $\left(\mathrm{T}_{2}\right)$, between 5 and 6 weeks after. Evaluators were blind to the condition of the participants. Except for one instrument, all of them were answered by the adult participating person. Moreover, an observational rating scale for the assessment of the interaction of the child with the adult was used.

\section{Alabama Parenting Questionnaire (APQ; Shelton et al., 1996)}

This is a self-report instrument where the mother or father self-assesses the frequency with which he/she shows certain behaviors toward the child. With adaptations, it has been shown to be a suitable instrument for preschool families (Clerkin et al., 2007; de la Osa et al., 2014). It has been shown to have good inter-observer reliability in Chilean families (Cova et al., 2017). Positive reinforcement (six items), parental involvement (seven items), and disciplinary inconsistency (four items) sub-scales were used. We broadened the scope of the last sub-scale by adding three items as follows: "If you don't get your child to obey you, finally you give up"; "One day you punish your child for doing something, but then, another day, you don't"; and "You set clear behavior rules for your child and make him/her respect them." We added these items on the basis that they increased Cronbach's alpha for the now seven item subscale, had high correlations with the original scale, and improved the scale in a confirmatory analysis. Each item has five response options $(1=$ not much to 5 = always). The internal consistencies of each sub-scale in the initial assessment were $0.52,0.69$, and 0.75 , respectively.

\section{Harsh Discipline Practice List (HDPL; Flores and Herrera, 2014)}

This 19-item scale measures harsh discipline behaviors, verbal maltreatment, and physical abuse and punishment. Each item has four response options ( $1=$ never to $4=$ always $)$. On the basis of an exploratory factor analysis, four items were removed because they affected reliability or cross-loaded. Two factors were identified: (1) physical punishment (nine items) and (2) humiliating treatment (six items). The internal consistency of each factor in the initial assessment was 0.77 and 0.63 , respectively.

\section{Keys to Interactive Parenting Scale (KIPS; Comfort et al., 2011)}

This is an observational instrument which evaluates the quality of 12 specific parental practices for caregivers of children aged 24-71 months. The interaction between the child and the caregiver in a 15-20 min play session is video recorded and afterward coded by a trained and accredited evaluator. Each parental practice is rated into five quality levels $(1=$ poor quality of parental practices to $5=$ good quality of parental practices). The global quality of parental practices is obtained by the average score of the 12 observed parenting practices. 
Psychometric studies show that the instrument has acceptable internal consistency and inter-judge agreement in its application (Inostroza et al., 2014). Five percent of these assessments were coded again by an experienced evaluator and a discrepancy of only $5.4 \%$ was seen. It showed an internal consistency of $\alpha=0.77$ in the initial assessment.

\section{Parental Evaluation Scale (PEP; Farkas-Klein, 2008)}

This is a self-administered measure to assess satisfaction and feelings of self-efficacy with regard to parenthood. It has a Likert format of six options ( $0=$ strongly disagree; $5=$ strongly agree). The factor analysis revealed one factor. Of its 10 original items, two were eliminated, resulting in an internal consistency of $\alpha=0.77$ in the initial assessment.

\section{Caregiver's Depressive Mood Scale (Rodríguez et al., 1996)}

The Caregiver's Depressive Mood Scale from the Behavioral and Socio-Emotional Problems Inventory was used. This instrument was developed in Chile to assess the mental health of preschoolers and potential risk factors. It includes four items with two response options (Yes $=1$ or $\mathrm{No}=0$ ) and had an internal consistency of $\alpha=0.61$ in the initial assessment.

\section{Eyberg Child Behavior Inventory (ECBI; Garcia-Tornal et al., 1998; Eyberg and Pincus, 1999)}

This is a 36-item instrument (each of them has seven response options from 1 = never to 7 = always), which uses parental report to assess behavioral problems in children aged between 2 and 16 . It has a behavioral problem scale with seven Likert format answer options, which measure the frequency of these problems, and an intensity scale with two response options (Yes/No), which assess the extent to which each problem is a concern for the informant. Both scales had internal consistencies of $\alpha=0.91$ in the initial assessment.

\section{Ethical Considerations}

This study was carried out in accordance with the recommendations of The International Ethical Guidelines for Biomedical Research Involving Human Subjects ${ }^{1}$, Research Ethics Committee of the University of Concepción, with written informed consent in accordance with the Declaration of Helsinki. The protocol was approved by the Research Ethics Committee of the University of Concepción.

\section{Data Analysis}

In order to avoid bias due to attrition, multiple imputation procedures were used; specifically, the Markov chained equation method implemented in the mice statistics library in R.

For the main analysis comparing the experimental and control groups, a multilevel approach was used, through generalized mixed linear models, using the lme4 library in R. An approach similar to analysis of covariance (ANCOVA) was used. The dependent variable was the result in the post-test. Group

${ }^{1}$ https://cioms.ch/wp-content/uploads/2017/01/WEB-CIOMS-EthicalGuidelines. pdf membership was a fixed effect, controlling for pre-test value, sex, and age of the child. Center was considered as a nested random effect in intercept-slope models. To determine the likelihood ratio test results over imputed databases, the Wald test adapted to multiple imputation databases, called Dm, which is approximately $F$ distributed, was used. The effect size was determined using the adaptation of Cohen's $d$ for ANCOVA (Cooper et al., 2009).

The analysis of the intervention results was carried out on an intention-to-treat basis (all participants assigned to the experimental condition are included regardless their attendance), as well as per-protocol, defined as attendance to three or more sessions. For this analysis, attenders for three or more sessions were compared to the participants from the control condition.

To identify possible moderators, interactions between the effects of the treatment and the pre-test values were analyzed. When the outcomes were significant, a region of significance analysis was performed, in order to identify pre-test values where treatment showed statistically significant results (Jaccard and Turrisi, 2003).

\section{RESULTS}

Considerable variability was seen in the attendance in the group receiving the program. Sixty one of the parents assigned to the experimental group (34\%) did not participate in any of the sessions, while 117 parents attended at least one session $(66 \%)$. Of these, 30 parents (17\% of the initial total) participated in one or two sessions and 87 parents $(49 \%$ of the initial total) participated in three or more sessions. Postassessment was possible with $84.4 \%$ of the participants in the experimental group and $92.8 \%$ of those in the control group. Regarding KIPS, it was possible to re-evaluate $82.6 \%$ of the experimental and $89.9 \%$ of the control group. Intraclass correlation coefficients seen in the initial measurements ranged between 0.01 for parental involvement and 0.09 for concern for behavior problems.

\section{Effects of the Intervention}

Tables 1, 2 show the values from the experimental and control groups for both pre and post measurements.

Table 3 shows the analysis of the main effects. Intentionto-treat analysis showed a decrease in physical punishment practices, $d=-0.37, F(1,176.4)=20.3, p<0.001$; and an increase in parental involvement, $d=0.23, F(1,369.2)=7.26, p=0.007$ as well as a decrease in the frequency of behavioral problems, $d=-0.35, F(1,908.4)=18.2, p<0.001$; and concern about them, $d=0.20, F(1,2112)=6.88, p=0.009$. The per-protocol analysis showed greater effect sizes except in the last case, where it remained almost identical: physical punishment practices, $d=-0.45, F(1,366,2)=24.3, p<0.001$; parental involvement, $d=0.35, F(1,191.9)=10.2, p=0.002$; behavioral problems, $d=-0.40, F(1,2610)=15.3, p<0.001$; parental concern for behavioral problems, $d=0.19, F(1,6851)=4.04, p=0.045$. Per-protocol analysis also showed an increase in the observed parental practices, $d=0.37, F(1,539.1)=8.7, p=0.003$. 
TABLE 1 | Anova results of pre and post evaluation in experimental group.

\begin{tabular}{|c|c|c|c|c|c|c|c|}
\hline & \multicolumn{2}{|c|}{ Pre $\left(T_{1}\right)$} & \multicolumn{2}{|c|}{ Post $\left(\mathrm{T}_{2}\right)$} & \multirow[b]{2}{*}{$p^{a}$} & \multirow[b]{2}{*}{$F(d f 1, d f 2)$} & \multirow[b]{2}{*}{ est $\eta^{2} G^{b}$} \\
\hline & Mean & $S D$ & Mean & $S D$ & & & \\
\hline Positive reinforcement & 4.66 & 0.35 & 4.71 & 0.35 & 0.090 & $2.88(1,5588.26)$ & 0.005 \\
\hline Parental involvement & 4.39 & 0.52 & 4.52 & 0.46 & $<0.001^{*}$ & $15.06(1,745.62)$ & 0.017 \\
\hline Disciplinary inconsistency & 2.48 & 0.83 & 2.23 & 0.78 & $<0.001^{*}$ & $18.90(1,146.05)$ & 0.025 \\
\hline Humiliating treatment & 0.06 & 0.21 & 0.04 & 0.13 & 0.180 & $1.80(1,5162.47)$ & 0.002 \\
\hline Physical punishment & 1.01 & 0.50 & 0.77 & 0.40 & $<0.001^{*}$ & $66.11(1,881.24)$ & 0.073 \\
\hline Observed parental practices & 3.77 & 0.78 & 3.71 & 0.78 & 0.436 & $0.61(1,424.23)$ & 0.002 \\
\hline Parental satisfaction ${ }^{\mathrm{C}}$ & 1.61 & 0.95 & 1.46 & 0.92 & $0.010^{*}$ & $6.61(1,2082.58)$ & 0.007 \\
\hline Depressed mood & 0.87 & 0.54 & 0.76 & 0.54 & $0.003^{*}$ & $8.96(1,288.60)$ & 0.010 \\
\hline Behavioral problems & 3.16 & 0.85 & 2.76 & 0.85 & $<0.001^{*}$ & $65.11(1,972.74)$ & 0.056 \\
\hline Parental concern for behavioral problems ${ }^{c}$ & 1.64 & 0.21 & 1.73 & 0.22 & $<0.001^{*}$ & $33.54(1,293.99)$ & 0.040 \\
\hline
\end{tabular}

${ }^{a}$ Anova $p$-value. ${ }^{b}$ Squared generalized eta. ${ }^{c}$ Reverse scoring scale. ${ }^{*}$ The difference between the mean scores is statistically significant $p<0.05$.

TABLE 2 | Anova results of pre and post evaluation in control group.

\begin{tabular}{|c|c|c|c|c|c|c|c|}
\hline & \multicolumn{2}{|c|}{ Pre $\left(T_{1}\right)$} & \multicolumn{2}{|c|}{ Post $\left(T_{2}\right)$} & \multirow[b]{2}{*}{$p^{a}$} & \multirow[b]{2}{*}{$F(d f 1, d f 2)$} & \multirow[b]{2}{*}{ est $\eta^{2} G^{b}$} \\
\hline & Mean & $S D$ & Mean & $S D$ & & & \\
\hline Parental involvement & 4.35 & 0.53 & 4.35 & 0.52 & 0.949 & $0.00(1,543.78)$ & 0.001 \\
\hline Disciplinary inconsistency & 2.51 & 0.77 & 2.42 & 0.74 & 0.103 & $2.66(1,1676.26)$ & 0.003 \\
\hline Humiliating treatment & 0.07 & 0.14 & 0.06 & 0.12 & 0.430 & $0.62(1,1080.33)$ & 0.002 \\
\hline Parental satisfaction ${ }^{c}$ & 1.70 & 0.87 & 1.57 & 0.90 & $0.031 *$ & $4.70(1,1165.60)$ & 0.006 \\
\hline Depressed mood & 0.88 & 0.48 & 0.80 & 0.54 & $0.026^{*}$ & $5.00(1,259.30)$ & 0.006 \\
\hline Behavioral problems & 3.20 & 0.81 & 3.09 & 0.89 & $0.036^{*}$ & $4.38(1,4431.67)$ & 0.005 \\
\hline Parental concern for Behavioral problems ${ }^{c}$ & 1.59 & 0.20 & 1.63 & 0.23 & $0.001^{*}$ & $7.02(1,2150.25)$ & 0.007 \\
\hline
\end{tabular}

${ }^{a}$ Anova $p$-value. ${ }^{b}$ Squared generalized eta. ${ }^{c}$ Reverse scoring scale. ${ }^{*}$ The difference between the mean scores is statistically significant $p<0.05$.

TABLE 3 | Results and effect comparison by group.

\begin{tabular}{|c|c|c|c|c|c|c|c|c|}
\hline & \multicolumn{3}{|c|}{ Intention to treat } & \multicolumn{3}{|c|}{ Per protocol } & \multicolumn{2}{|c|}{ Adjusted effect for both groups ${ }^{a}$} \\
\hline Parental involvement & 7.260 & $0.007^{*}$ & 0.227 & 10.200 & $0.002^{*}$ & 0.345 & 0.059 & $0.015^{*}$ \\
\hline Disciplinary inconsistency & 2.400 & 0.121 & -0.139 & 4.380 & 0.036 & -0.227 & 0.607 & 0.218 \\
\hline Humiliating treatment & 0.588 & 0.443 & -0.070 & 1.360 & 0.243 & -0.142 & 1.000 & 0.722 \\
\hline Parental satisfaction ${ }^{d}$ & 0.276 & 0.600 & -0.051 & 1.380 & 0.241 & -0.130 & 1.000 & 0.722 \\
\hline Depressed mood & 0.553 & 0.457 & -0.067 & 0.603 & 0.438 & -0.079 & 1.000 & 0.722 \\
\hline Behavioral problems & 18.200 & $<0.001^{*}$ & -0.348 & 15.300 & $<0.001^{*}$ & -0.398 & $<0.001^{*}$ & $<0.001^{*}$ \\
\hline Parental concern for behavioral problems ${ }^{d}$ & 6.880 & 0.009* & 0.202 & 4.040 & $0.045^{*}$ & 0.186 & 0.061 & 0.223 \\
\hline
\end{tabular}

a Multiple comparisons analysis using Bonferroni-Holm correction method. ${ }^{b} \mathrm{~F}$ distribution for ratio likelihood test through Wald test adapted for multiple imputations. ${ }^{c}$ Effect size. ${ }^{d}$ Reverse scoring scale. * The difference between the mean scores is statistically significant $p<0.05$.

Moderation analysis for intention-to-treat analysis was significant only for humiliating treatment, $F(1,839)=10.18$, $p=0.001$, est $\eta^{2} G=0.03$ indicating that the program was effective in reducing these behaviors in those parents having higher initial levels (pre-test values on humiliating treatment greater than 0.84 standard deviations below the mean). Per-protocol, moderation was significant for positive reinforcement, $F(1,25,587)=5.5, p=0.019$, est $\eta^{2}{ }_{G}=0.02$, 
and parental involvement, $F(1,6679)=5.081 ; p=0.024$, est $\eta^{2}{ }_{G}=0.02$. For both variables, greater effects of the program were seen in those parents with lower initial values of positive reinforcement and parental involvement with their children (for positive reinforcement, the treatment was effective when pre-test values were lower than -0.06 standard deviations below the mean, and for parental involvement, for values lower than 0.74 standard deviations above the mean).

\section{DISCUSSION}

The purpose of this study was to assess the effectiveness of a brief parenting training program universally applied in families of preschool children. Although the program encouraged diverse members of the family group to participate, in practice, in our socio-cultural context, those who participate in these activities are almost only mothers.

The results are promising. At the conclusion of the program, positive changes in the expected direction in parenting practices and a decrease in the children's behavioral problems were seen. Intention-to-treat analysis showed a decrease in humiliating treatment and physical punishment practices, and an increase in parental involvement, as well as a decrease in the frequency of behavior problems in the children and the concern about them on the part of the parents. The intention-to-treat analysis is the only approach that respects the initial randomization and, therefore, the only one that discounts the possibility that differences seen between the experimental and control groups are due to differential attrition between the groups (Kraemer, 2015). However, in this type of analysis, the magnitude of the effect size is diminished by the inclusion of people who, having said they will participate, subsequently do not. In effect, it assesses the benefit of the offer of training, which is the most clinically relevant where this training to be scaled up. In this study, a third of the participants did not take up the offer of training, a rate comparable to similar studies, particularly in promotional or preventative programs ( Simkiss et al., 2013; Chacko et al., 2016). This, together with participants attending few sessions or irregularly, weakens the ability to detect the direct effects of the intervention.

Other factors making the detection of effects in the universal parenting training programs more difficult are the so-called floor and ceiling effects, since many parents showed suitable parenting practices prior to the intervention and many children did not show behavioral problems. Therefore, it is more difficult to demonstrate changes; thus, parenting training programs have more evident effects in those showing higher levels of problems in the initial assessment (Lundahl et al., 2008; Proctor and Brestan-Knight, 2016). We saw this in the current study, where greater effects of the program were seen in those parents with lower initial values of positive reinforcement and involvement practices with their children; importantly, in the intention-to-treat analysis, an effect of moderation was observed in relation to humiliating behaviors, indicating that the program was effective in reducing these behaviors in those parents having higher initial levels at the outset. However, the effect of floor and ceiling effects was to reduce the magnitude of the main effects of treatment.

Additionally, other effects were seen in the per-protocol analysis, specifically improved parental practices during play interactions seen in the observational data. However, given that per-protocol analyses are not balanced by the original randomization, we cannot rule out possible biases arising from participant characteristics or other factors.

This study has several limitations. First, we were unable to assess whether the favorable changes seen persisted in the longer term. Second, in spite of the universal nature of the offered program, many of the parents in the experimental group who could have benefitted from it did not participate (either refusing the invitation or not attending any of the sessions). This is particularly important for universal promotional and preventive parenting programs when some families may lack motivation to participate. In general, the main barriers to participation were time commitments and/or an incompatibility with other work or household responsibilities (Chacko et al., 2016). Considering these usual barriers, the percentage of participation was significant. We think our modification of the previous version of the Day by Day Program encouraged participation by offering fewer sessions as well as increased involvement of educational personnel in the participating centers.

A third limitation of our study is the low values of internal consistency for most of our measurements, with the exception of the Eyberg Questionnaire. Three measurements were too low (positive reinforcement, depressive mood, and humiliating treatment) and the result related to them must be interpreted with care. The Alabama Questionnaire is, however, one of the best self-report instruments to assess parental practices, and it has shown good psychometrics in the same type of population as this study (Scott et al., 2011; Cova et al., 2017).

Another limitation of this study is that its main results mainly depended on reports of the parents about their parenting practices as well as the children's behavior. It is difficult to overcome this limitation. The assessment of the children's behavior in a school context has been shown to have limitations and, in fact, is generally not shown to be very sensitive to the effects of parenting programs (Scott and Gardner, 2015). Thus, one of the strengths of this study was our use of an observational measure, which showed positive effects for the intervention in the per-protocol analysis. Finally, sample size was too small to assess relevance of age and sex differences in intervention's response. Best interventions are those that specify for whom the treatment might be most effective. Future studies could elucidate any differences based on these possible moderators (Kraemer et al., 2002).

In countries like Chile, undesirable parenting behaviors, such as inappropriate treatment and physical punishment, are widely used (Vizcarra et al., 2001). Structured, replicable, evidence-based programs, based on social learning theory and encouragement of parent-child links, are a promising strategy for reducing these behaviors and need to be available in middle and low income countries (Knerr et al., 2013; Webster-Stratton and Herman, 2015). 


\section{CONCLUSION}

Our results demonstrate the effectiveness of this universal brief training program designed to improve parenting practices and decrease problematic behavior in children in a middle income country.

\section{AUTHOR CONTRIBUTIONS}

PR designed, developed, and implemented the parental training program and contributed to data collection. FC conducted study design, literature search, data interpretation, and wrote the manuscript. SS contributed to study design, program implementation, and data collection. CB performed the statistical

\section{REFERENCES}

Bayer, J. K., Hiscock, H., Ukoumunne, O. C., Scalzo, K., and Wake, M. (2010). Three-year-old outcomes of a brief universal parenting intervention to prevent behaviour problems: randomised controlled trial. Arch. Dis. Child. 95, 187-192. doi: 10.1136/adc.2009.168302

Chacko, A., Jensen, S. A., Lowry, L. S., Cornwell, M., Chimklis, A., Chan, E., et al. (2016). Engagement in behavioral parent training: review of the literature and implications for practice. Clin. Child Fam. Psychol. Rev. 19, 204-215. doi: 10.1007/s10567-016-0205-2

Clerkin, S. M., Marks, D. J., Policaro, K. L., and Halperin, J. M. (2007). Psychometric properties of the alabama parenting questionnairepreschool revision. J. Clin. Child Adolesc. Psychol. 36, 19-28. doi: 10.1207/ s15374424jccp3601-3

Comfort, M., Gordon, P. R., and Naples, D. (2011). KIPS: an evidence-based tool for assessing parenting strengths and needs in diverse families. Infants Young Child. 24, 56-74. doi: 10.1097/Iyc.0b013e3182001bd3

Cooper, H. M., Hedges, L. V., and Valentine, J. C. (2009). The Handbook of Research Synthesis and Meta-Analysis, 2nd Edn. New York, NY: Russell Sage Foundation.

Cova, F., Bustos, C., Rincon, P., Streiner, D., Grandon, P., Saldivia, S., et al. (2017). "Psychometric properties of the alabama parenting questionnaire adapted to families of chilean preschoolers" by Cova, Felix; Bustos, Claudio; Rincon, Paulina; Streiner, David; Grandon, Pamela; Saldivia, Sandra; Inostroza, Carolina, has been successfully submitted online and is presently being given full consideration for publication in infant mental health journal. Infant Ment. Health J. 38, 249-257. doi: 10.1002/imhj.21631

de la Osa, N., Granero, R., Penelo, E., Domenech, J. M., and Ezpeleta, L. (2014). Psychometric properties of the Alabama Parenting Questionnaire-Preschool Revision (APQ-Pr) in 3 year-old Spanish preschoolers. J. Child Fam. Stud. 23, 776-784. doi: 10.1007/s10826-013-9730-5

Eyberg, S., and Pincus, D. (1999). ECBI \& SESBI-R : Eyberg Child Behavior Inventory and Sutter-Eyberg Student Behavior Inventory-Revised : Professional Manual. Odessa, FL: Psychological Assessment Resources.

Farkas-Klein, C. (2008). Parental Evaluation Scale (EEP): development, psychometric properties and applications. Univ. Psychol. 7, 457-467.

Flores, J. J., and Herrera, L. M. F. (2014). Design and psychometric validation of the Harsh Discipline Practice List. Rev. Rev. Iberoam. Diagn. Ev. 2, 137-153.

Forehand, R., Lafko, N., Parent, J., and Burt, K. B. (2014). Is parenting the mediator of change in behavioral parent training for externalizing problems of youth? Clin. Psychol. Rev. 34, 608-619. doi: 10.1016/j.cpr.2014.10.001

Furlong, M., McGilloway, S., Bywater, T., Hutchings, J., Smith, S., and Donnelly, M. (2013). Are group-based parenting programmes effective in the treatment of childhood conduct problems? A Cochrane review. Eur. Child Adolesc. Psychiatry 22, S196-S196.

Garcia-Tornal, S., Calzada, E., Eyberg, S., Mas, J., Vilamala, C., Baraza, C., et al. (1998). Inventario Eyberg del comportamiento en niños. Normalización de analysis and contributed to data interpretation. PG participated in the design, development, and implementation of the program. CI conducted data collection and participated in article edition. DS participated in data interpretation and writing. VB participated in data collection and article edition. MK contributed to study design, data interpretation, and writing. All authors read and approved the final manuscript.

\section{FUNDING}

This study was a part of the projects FONDEF ID14I10058 and FONDECYT 1120716 of the Chilean National Commission for Scientific and Technological Research (Comisión Nacional de Investigación Científica y Tecnológica, CONICYT).

la versión española y su utilidad para el pediatra extrahospitalario. An. Esp. Pediatr. 48, 475-482.

Gardner, F., Connell, A., Trentacosta, C. J., Shaw, D. S., Dishion, T. J., and Wilson, M. N. (2009). Moderators of outcome in a brief family-centered intervention for preventing early problem behavior. J. Consult. Clin. Psychol. 77, 543-553. doi: $10.1037 / \mathrm{a} 0015622$

Herrman, H., Saxena, S., and Moodie, R. (2005). Promoting Mental Health : Concepts, Emerging Evidence, Practice. Geneva: World Health Organization.

Hiscock, H., Bayer, J. K., Price, A., Ukoumunne, O. C., Rogers, S., and Wake, M. (2008). Universal parenting programme to prevent early childhood behavioural problems: cluster randomised trial. Br. Med. J. 336, 318-321. doi: 10.1136/bmj. 39451.609676.AE

Inostroza, C., Contreras, G., Cova, F., Rincón, P., Grandón, P., and Saldivia, S. (2014). Acuerdo interjueces en el empleo de la escala observacional de prácticas parentales KIPS en una muestra de madres/niños chilenos de 36 a 71 meses. Ajayu 12, 120-134.

Jaccard, J., and Turrisi, R. (2003). Interaction Effects in Multiple Regression, 2nd Edn. Thousand Oaks, CA: Sage Publications. doi: 10.4135/9781412984522

Kaminski, J. W., Valle, L. A., Filene, J. H., and Boyle, C. L. (2008). A meta-analytic review of components associated with parent training program effectiveness. J. Abnorm. Child Psychol. 36, 567-589. doi: 10.1007/s10802-007-9201-9

Kato, N., Yanagawa, T., Fujiwara, T., and Morawska, A. (2015). Prevalence of children's mental health problems and the effectiveness of populationlevel family interventions. J. Epidemiol. 25, 507-516. doi: 10.2188/jea.JE2014 0198

Klahr, A. M., and Burt, S. A. (2014). Practitioner review: evaluation of the known behavioral heterogeneity in conduct disorder to improve its assessment and treatment. J. Child Psychol. Psychiatry 55, 1300-1310. doi: 10.1111/jcpp.12268

Knerr, W., Gardner, F., and Cluver, L. (2013). Improving positive parenting skills and reducing harsh and abusive parenting in low- and middle-income countries: a systematic review. Prev. Sci. 14, 352-363. doi: 10.1007/s11121-0120314-1

Kraemer, H. (2015). "Evaluating interventions," in Ruttert's Child and Adolescent Psychiatry, eds A. Thaper, D. Pine, J. Leckman, S. Scott, M. Snowling, and E. Taylor (Oxford: Wiley), 177-187. doi: 10.1002/9781118381953.ch14

Kraemer, H. C., Wilson, G. T., Fairburn, C. G., and Agras, W. S. (2002). Mediators and moderators of treatment effects in randomized clinical trials. Arch. Gen. Psychiatry 59, 877-883. doi: 10.1001/archpsyc.59.10.877

Lundahl, B. W., Tollefson, D., Risser, H., and Lovejoy, M. C. (2008). A metaanalysis of father involvement in parent training. Res. Soc. Work Pract. 18, 97-106. doi: 10.1177/1049731507309828

Menting, A. T. A., de Castro, B. O., and Matthys, W. (2013). Effectiveness of the incredible years parent training to modify disruptive and prosocial child behavior: a meta-analytic review. Clin. Psychol. Rev. 33, 901-913. doi: 10.1016/ j.cpr.2013.07.006

Merry, S., and Moor, S. (2015). "School-based mental health interventions," in Ruttert's Child and Adolescent Psychiatry, eds A. Thaper, D. Pine, J. Leckman, S. Scott, M. Snowling, and E. Taylor (Oxford: Wiley), 545-558. 
Rincón et al.

Positive Parental Practices Program Trial

Offord, D. R. (2000). Selection of levels of prevention. Addict. Behave. 25, 833-842. dor: 10.1016/S0306-4603(00)00132-5

Proctor, K. B., and Brestan-Knight, E. (2016). Evaluating the use of assessment paradigms for preventive interventions: a review of the triple $\mathrm{p}$ - positive parenting program. Child. Youth Serve. Rev. 62, 72-82. do: 10.1016/j.childyouth. 2016.01.018

Rodríguez, S., Lira, M., Arancibia, V., and Bralic, S. (1996). Inventario de Problemas Conductuales y Socioemocionales: 3-5 Años. Santiago: Ediciones Universidad Católica.

Scott, S., Briskman, J., and Lads, M. R. (2011). Measuring parenting in community and public health research using brief child and parent reports. J. Child Fam. Stud. 20, 343-352. doa: 10.1007/s10826-010-9398-z

Scott, S., and Gardner, F. (2015). "Parenting Programs," in Ruttert's Child and Adolescent Psychiatry, eds A. Thaper, D. Pine, J. Leckman, S. Scott, M. Snowling, and E. Taylor (Oxford: Wiley), 483-495. doe: 10.1002/9781118381953.ch37

Shelton, K. K., Erick, P. J., and Wotton, J. (1996). Assessment of parenting practices in families of elementary school-age children. J. Chin. Child Psychol. 25, 317-329. do: 10.1207/s15374424jccp2503_8

Simkiss, D. E., Snooks, H. A., Stallard, N., Kimani, P. K., Sewell, B., Fitzsimmons, D., et al. (2013). Effectiveness and cost-effectiveness of a universal parenting skills programme in deprived communities: multicentre randomised controlled trial. BMJ Open 3:e002851. doi: 10.1136/bmjopen-2013-002851

Smedler, A. C., Hern, A., Wiklund, S., Anttila, S., and Pettersson, A. (2015). Programs for prevention of externalizing problems in children: limited evidence for effect beyond 6 months post intervention. Child Youth Care Forum 44, 251-276. doe: 10.1007/s10566-014-9281-y

Streiner, D. L. (2002). The two Es of research: efficacy and effectiveness trials. Can. J. Psychiatry 47, 552-556. doa: 10.1177/070674370204700607

Vizcarra, M. B., Cortes, J., Bustos, L., Alarcon, M., and Munoz, S. (2001). Child abuse in Temuco, Chile. Prevalence and risk factors. Rev. Med. Chile 129, 1425-1432.

Webster-Stratton, C., and Herman, K. C. (2015). Disseminating incredible years series early-intervention programs: integrating and sustaining services between school and home (vol 47, pg 36, 2010). Psychol. Sch. 52, 1051-1051.

Conflict of Interest Statement: The authors reports grants from CONICYT (Chilean National Commission for Scientific and Technological Research): This study was financed by the FONDEF ID14I10058. In addition, authors have a patent A-273006 issued to Universidad de Concepcion: "Día a Día UdeC(c)" Parental Training Program was developed by Universidad de Concepcion.

Copyright (c) 2018 Rincón, Cove, Saldivia, Bustos, Grandón, Inostroza, Strainer, Bühring and King. This is an open-access article distributed under the terms of the Creative Commons Attribution License (CC BY). The use, distribution or reproduction in other forums is permitted, provided the original authors) and the copyright owners) are credited and that the original publication in this journal is cited, in accordance with accepted academic practice. No use, distribution or reproduction is permitted which does not comply with these terms.

Frontiers in Psychology | www.frontiersin.org

9

September 2018 | Volume 9 | Article 1751 